polymorphisms in the T2R38 (bitter taste) receptor in response to chemical ligands, which is predicted to lead to changes in $\mathrm{CBF}$, which are transient and small in magnitude. These receptors are of interest as they have been shown to 'sense' quorum sensing molecules produced by Pseudomonas aeruginosa (Pa) and may thus be disease modifiers in patients with cystic fibrosis. This work describes the development of a rapid CBF assay with sufficient sensitivity to provide a read-out of airway epithelial cell responses to stimulation in vitro.

Air-liquid interface (ALI) cultures were obtained from surplus clinical diagnostic samples. Cells in ALI were exposed to phosphate buffered saline (PBS; negative control) and adenosine 5'triphosphate (ATP; positive control). Experiments were conducted in temperature-controlled wells under a $40 \times$ light microscope. Cilia were imaged by high-speed video camera and CBF expressed as a ratio of stimulated/basal frequencies.

CBF increased in ALI cultures exposed to ATP $(n=4)$ but not PBS $(\mathrm{n}=3)$, in experiments at $24-25^{\circ} \mathrm{C}$; this effect was not seen at $37^{\circ} \mathrm{C}$. Mean \pm SEM stimulated/basal CBF ratio was 1.00 \pm 0.05 in the PBS-exposed cells, and $1.31 \pm 0.08$ in the ATPexposed cells $(p=0.029)$. Inter-observer variability $(n=2)$ was lower than within-sample CBF variability (95\% limits of agreement from -0.66 to $1.62 \mathrm{~Hz}$ ). Intra-observer variability was good with $95 \%$ limits of agreement between -0.31 to $0.52 \mathrm{~Hz}$.

An assay has been developed to detect rapid changes in $\mathrm{CBF}$ in ALI cultures using ATP as a positive control. Further work is being undertaken to a) optimise this assay in epithelial cells in suspension, thus increasing throughput, and b) assess more relevant chemicals and culture media. Once optimised, this assay will be used to study the effects of Pa quorum sensing molecules on ciliated epithelial cells in vitro, from patients of varying TAS2R38 genotypes.

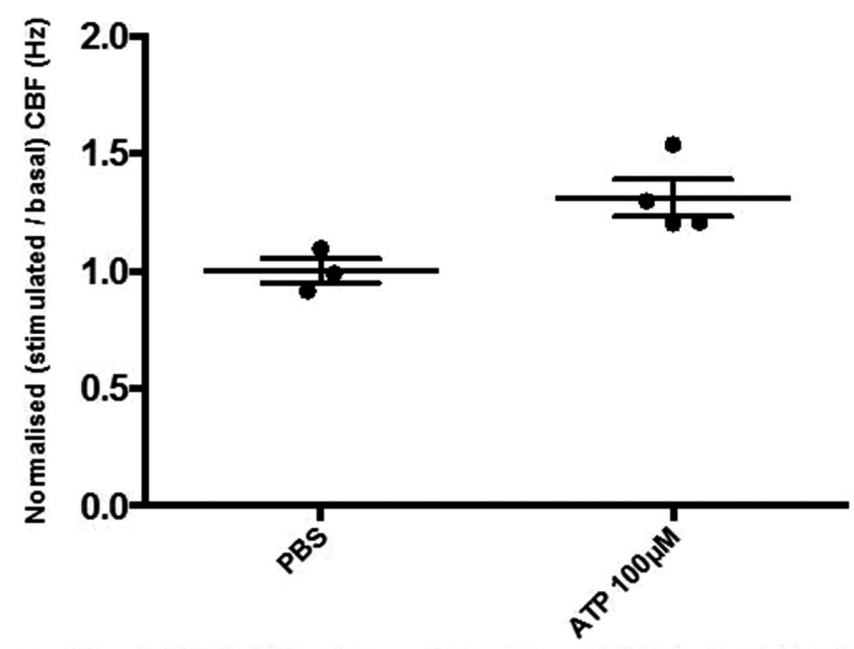

Ratio of stim ulated/basal CBF in ALI cultures exposed first to PBS, followed by PBS ( $n=3$ cultures) or A TP $100 \mu M$ ( $n=4$ cultures).

Abstract S69 Figure 1

\section{S70 EXPERIMENTAL HUMAN PNEUMOCOCCAL COLONISATION IS AN ASYMPTOMATIC EVENT IN HEALTHY ADULTS}

${ }^{1}$ A Trimble, ${ }^{1}$ AM Collins, ${ }^{1}$ CA Hancock, 'SB Gordon, ${ }^{1}$ DM Ferreira, ${ }^{2}$ AD Wright. 'Liverpool School Tropical Medicine, Liverpool, UK; ${ }^{2}$ Local Comprehensive Research Network, Liverpool, UK

10.1136/thoraxjnl-2015-207770.76
Background Pneumococcal colonisation is a necessary precursor for pneumococcal diseases. Previous studies have suggested that pneumococcal colonisation in children is symptomatic and that there is a relationship between symptom severity/frequency and colonisation density. The literature refers to colonisation in adults as an asymptomatic event but no studies have used robust methodology. Using the Experimental Human Pneumococcal Colonisation (EHPC) model, we investigated whether pneumococcal colonisation (or co-colonisation with a respiratory virus) is symptomatic in healthy adults.

Methods Non-smoking healthy adults aged 18-60 years old were recruited and inoculated intranasally with $0.1 \mathrm{ml}$ per naris of pneumococcus (serotype 6B, 23F) or saline (control). Nasal and non-nasal symptoms were monitored pre-inoculation and for 7 days post-inoculation using a modified Likert score. Symptom severity scoring ranged from 1 (none) to 7 (cannot function). Area under the curve (AUC) was calculated for each participant. Mean values were compared using ANOVA between the groups. Results Data from 53 participants were analysed. 45 were inoculated with pneumococcus and 8 with saline. In total, 14 became experimentally colonised. Colonised and non-colonised participants reported similar symptom severity scores (Figure 1). Mean severity scores for both nasal and non-nasal symptoms did not significantly differ according to colonisation status ( $p>0.05$ ), nor was there any significant association with any particular symptom. In the 14 experimentally colonised participants, density of colonisation did not correlate with symptom severity scores $\left(\mathrm{R}^{2}=0.03, \mathrm{p}=0.89\right) .6$ participants were co-colonised (with pneumococcus and a respiratory virus), although they showed a trend towards experiencing more non-nasal symptoms than other groups, their mean symptom severity was still occasional only $(\mathrm{p}>0.05)$.

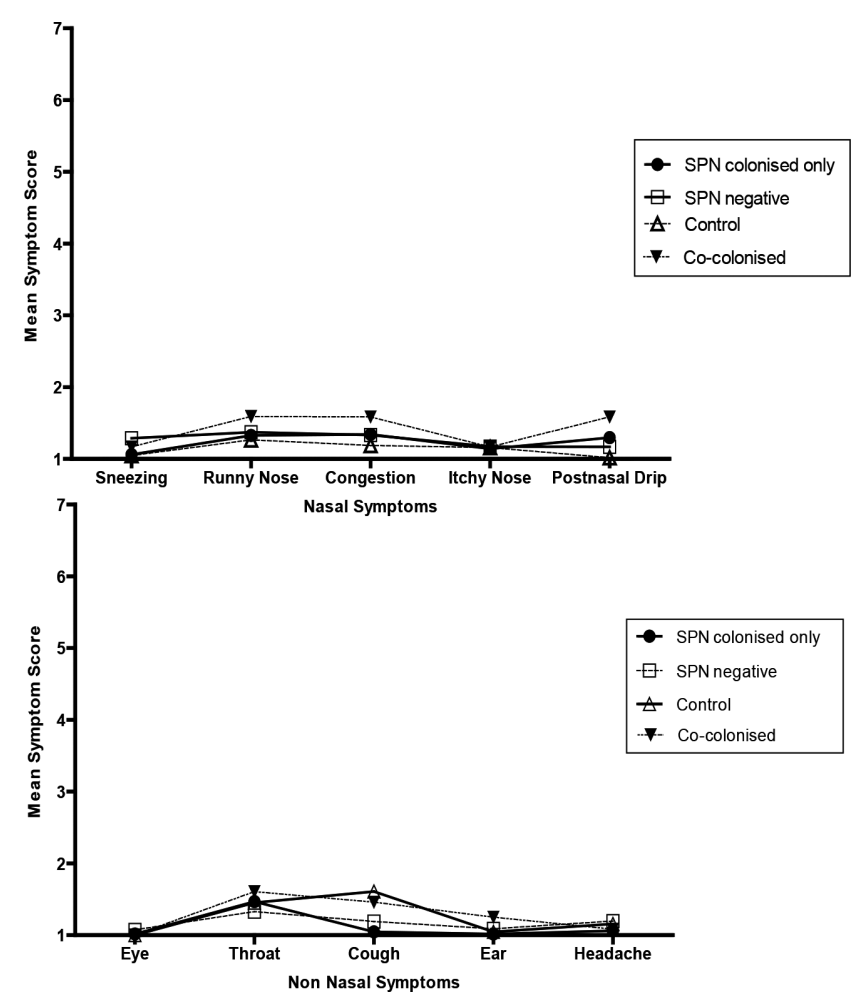

Abstract S70 Figure 1 Mean Symptom Scores for Nasal and Nonnasal Symptoms. SPN = Streptococcus pneumoniae. Control $=$ saline. Co-colonised $=$ colonised with Streptococcus pneumoniae and virus 
Conclusion In healthy adults pneumococcal colonisation is an asymptomatic event, regardless of density or viral cocolonisation.

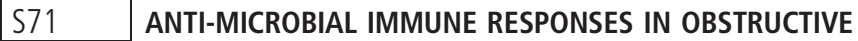 LUNG DISEASES}

${ }^{1} \mathrm{~F}$ Jaat, ${ }^{1} \mathrm{~S}$ Hasan, ${ }^{1} \mathrm{C}$ Lanyon, ${ }^{2} \mathrm{~A}$ De Soyza, ${ }^{1} \mathrm{~S}$ Todryk. ${ }^{1}$ Northumbria University, Newcastle Upon Tyne, UK; ${ }^{2}$ Freeman Hospital, Newcastle Upon Tyne, UK

\subsection{6/thoraxjnl-2015-207770.77}

Background People with chronic lung diseases, such as Bronchiectasis (BE) and smoking-induced Chronic Obstructive Pulmonary Disease (COPD), are susceptible to lung infections which can exacerbate their disease and can be life threatening. A relatively limited range of pathogens cause infections in these conditions and patients suffer repeated infections. It is unclear why such infections do not elicit protective adaptive immune responses. We wish to better understand immune responses against lung-infecting microbes in people with underlying lung disease since immune responses may be connected to disease pathology and also to protection from infection, and may provide a useful marker of colonisation.

Methods We took peripheral blood samples from $114 \mathrm{BE}$ and 47 COPD patients attending secondary care clinics, and 25 healthy controls, and extracted PBMC and serum. The patients were well-characterised clinically, including their history, aetiology, lung function and longitudinal microbial colonisation. T cell and antibody responses were measured against a panel of common lung-infecting microbial antigens (bacteria, fungi and viruses) using our in-house well-characterised assays (ELISA and ELIspot, respectively). These provided quantitative outputs of specific antibody titre and reactive gamma-interferon-secreting $\mathrm{T}$ cells per million PBMC, validated using positive controls. The sputum of patients was cultured, and microbial colonisation defined using prior definitions. Correlations between culture status and bacterial immune responses were analysed.

\begin{tabular}{|l|r|r|}
\hline \multicolumn{1}{|c|}{ Abstract S71 Table 1 } & \multicolumn{1}{l|}{ COPD } \\
\hline P.aeruginosa & 52.6 & 14.8 \\
\hline H.influenzae & 53.5 & 40.4 \\
\hline A. fumigatus & 23.6 & 4.2 \\
\hline M. catarrhalis & 39.4 & 29.7 \\
\hline S.pneumoniae & 47.3 & 21.2 \\
\hline S.maltophilia & 16 & 4.2 \\
\hline Candida & 15.7 & 8.5 \\
\hline S.aureus & 31.5 & 2.1 \\
\hline E.coli & 21 & 6.3 \\
\hline
\end{tabular}

Results The predominant pathogens varied between BE and COPD as expected (percentages in Table 1). These included
Pseudomonas, Haemophilus influenzae, Streptococcus pneumoniae and Moraxella $s p p$. We found that specific $\operatorname{IgG}$ antibody responses correlated with bacterial sputum culture data for Pseudomonas $(\mathrm{R}=0.61, \mathrm{p}=0.0001)$, but not with lung function nor number of exacerbations. In contrast, specific $\mathrm{T}$ cell responses did not correlate with microbiology.

Conclusions Our findings suggest that immune responses measured in the blood against potential lung pathogens contribute minimally to protection from infection or pathology. These tests may however help define colonisation status and could be used as surrogate markers of pathogens in the lung. The poor correlation between $\mathrm{T}$ cell responses may be a facet of the disease.

\section{Improving lung cancer outcomes}

\section{S72 IMPROVING LUNG CANCER SURVIVAL IN ENGLAND EVIDENCED THROUGH MULTIPLE DATA SOURCES}

${ }^{1} \mathrm{P}$ Beckett, ${ }^{1}$ I Woolhouse, ${ }^{2} \mathrm{~S}$ Walters, ${ }^{2} \mathrm{~S}$ Benitez-Majano, ${ }^{2} \mathrm{P}$ Muller, ${ }^{3} \mathrm{D}$ West, ${ }^{4} \mathrm{~S}$ McPhail, 4J Broggio, 'MD Peake. 'Royal College of Physicians, London, UK; ${ }^{2}$ Cancer Research UK Cancer Survival Group, London School of Hygiene and Tropical Medicine, London, UK; ${ }^{3}$ Society of Cardiothoracic Surgeons, Great Britain and Ireland; ${ }^{4}$ NHS Cancer Improvement, National Cancer Intelligence Network, London, UK

\subsection{6/thoraxjnl-2015-207770.78}

Introduction We have collated data from several sources to demonstrate that efforts made over the last 10 years to use data to drive service improvement and improved patient outcomes for UK patients with lung cancer have been successful.

Methods We used data submitted to the National Lung Cancer Audit (NLCA), National Cancer Registration Service (NCRS), Office for National Statistics (ONS) and Society of Cardiothoracic Surgeons (SCTS) from 1995-2013. We calculated numbers and proportions undergoing surgery, case-mix adjusted hazard ratios for death, and actual and predicted (using hybrid analyses) 1-year and 5-year survival for lung cancer patients in England. An international comparison has been made using data from the CONCORD-2 study.

Results In the NLCA, the proportion of NSCLC patients undergoing resection has risen from $14 \%$ (2005) to $23 \%$ (2013). Over this period, annual primary lung cancer resections have risen from 3,220 to 6,713 .

NLCA data, adjusted for age, sex, stage and PS, indicates a gradually falling hazard ratio for death (2013 HR 0.87, 95\% CI 0.85-0.89 compared to 2008). ONS data demonstrates a gradual improvement in both $1 \mathrm{yr}$ and $5 \mathrm{yr}$, and mirrors the increase in the number of resections carried out over the lifetime of the NLCA. Comparison of 1YS with other countries suggests that England has passed the survival measured in Denmark in 200407 (35\%), but still lags behind Canada (42\%) and Sweden $(46 \%)$. In another analysis using NCRS data, comparing stagespecific 1 yr survival in England in 2004-07 and 2012, improvements are most marked in patients with early stage disease.

Conclusion Whilst many changes have taken place in the management of lung cancer over the last 10 years, the close temporal association between the date of the first NLCA report (2005), the numbers of resections carried out and the significant improvements in 1 and $5 \mathrm{yr}$ survival (weighted towards earlier stages) and mortality we report here, would strongly suggest that the NLCA has been successful in its aim to improve standards of care and outcomes for patients. These improvements in survival 